\title{
Insights into the neuropathology of cerebral ischemia and its mechanisms
}

\begin{abstract}
Cerebral ischemia is a result of insufficient blood flow to the brain. It leads to limited supply of oxygen and other nutrients to meet metabolic demands. These phenomena lead to brain damage. There are two types of cerebral ischemia: focal and global ischemia. This condition has significant impact on patient's health and health care system requirements. Animal models such as transient occlusion of the middle cerebral artery and permanent occlusion of extracranial vessels have been established to mimic the conditions of the respective type of cerebral ischemia and to further understand pathophysiological mechanisms of these ischemic conditions. It is important to understand the pathophysiology of cerebral ischemia in order to identify therapeutic strategies for prevention and treatment. Here, we review the neuropathologies that are caused by cerebral ischemia and discuss the mechanisms that occur in cerebral ischemia such as reduction of cerebral blood flow, hippocampal damage, white matter lesions, neuronal cell death, cholinergic dysfunction, excitotoxicity, calcium overload, cytotoxic oedema, a decline in adenosine triphosphate (ATP), malfunctioning of $\mathrm{Na}+\mathrm{K}+-$ ATPase, and the blood-brain barrier breakdown. Altogether, the information provided can be used to guide therapeutic strategies for cerebral ischemia.
\end{abstract}

Keyword: Cerebral ischemia; Excitotoxicity; Neuropathology mechanisms; Stroke 